\title{
ARQUEOACÚSTICA MAYA. LA NECESIDAD DEL ESTUDIO SISTEMÁTICO DE EFECTOS ACÚSTICOS EN SITIOS ARQUEOLÓGICOS
}

\author{
Cliara Garzh
}

Instituto de Investigaciones en Matemáticas Aplicadas y en Sistemas, UNAM

Andrés Merina

Facultad de Ciencias, UNAM

Pablo Padil.LA

Instituto de Investigaciones en Matemáticas Aplicadas y en Sistemas, UNAM

Alejandro Rasos

Facultad de Ciencias, UNAM

Francisca Zalaquett

Instituto de Investigaciones Antropológicas, UNAM

Ésta es la relación de cómo todo estaba en suspenso, todo en calma [... Legó aquí entonces La palabra, vinieron Tepeu y Kukumatz en la oscuridad, en la noche y hablaron entre si $|\ldots|$

Popol Vin

\section{Introducción}

La gran cantidad de efectos acústicos en sitios arqueológicos de diversas culturas pone de manifiesto la necesidad de sistematizar su estudio, no sólo desde el punto de vista estrictamente fisico y arquitectónico, sino con una perspectiva más amplia, tratando de entenderlos como parte del contexto antropológico. La posibilidad de correlacionar estos efectos con otros aspectos de carácter cultural

\footnotetext{
"Agradecemos la información histónica brindada por la doctora Carmen Valverde, el apoyo computacional de Ana Pèrez Arteaga y Ramiro Châvez y al proyecto L/47899.F de CONACYT.
} 
puede ser de gran utilidad para conocer el diseño y funcionalidad (no sỏlo estrictamente religiosa, sino política, comercial, etc.) de diversas construcciones prehispánicas. Dentro de este enfoque, es indispensable investigar hasta qué punto los constructores estaban conscientes de estos fenómenos y si los incorporaron en su diseño con la intención de aprovechar los edificios en rituales u otras manifestaciones de carácter colectivo o privado. Desde nuestro punto de vista, este enfoque proporciona una linea de investigación relevante que podria complementar y enriquecer otras, constituyendo una herramienta adicional. Para realizar el estudio de los efectos acústicos, mencionados es imprescindible el esclarecimiento de los mecanismos fisicos subyacentes. En este proceso surge de forma natural la necesidad de formular modelos matemáticos y de implementarlos numéricamente mediante el uso de computadoras.

Este trabajo está organizado de la manera siguiente: después de una introducción general, en la sección 1 se presenta una discusión sobre lo que consideramos deberia constituir un marco básico para desarrollar un enfoque arqueoacistico; en la sección 2 abordamos los antecedentes de trabajos sobre acústica prehispánica en la zona maya; la sección 3 comprende algunos aspectos relevantes de las manifestaciones escénicas mayas que muestran que los mayas estaban conscientes de la acústica; en la sección 4 discutimos varios fenómenos acústicos, y en la 5 , nos concentramos especificamente en el caso de la cola del quetzal en Chichén Itzá. Este fenómeno consiste en que el eco de un aplauso frente a la escalera de El Castillo se escucha no como un "rebote" único, sino como un eco barrido que se asemeja al canto de un pájaro. Tal efecto ha sido objeto de estudios cuantitativos detallados utilizando básicamente los métodos que podriamos llamar de acústica geométrica (o de rayos), en analogía con los métodos de la óptica geométrica. Estos métodos explican la selección de las frecuencias que constituyen el barrido. pero no proporcionan una descripción dinámica (en tiempo real) del fenómeno. Partimos de aqui para plantear un modelo de frentes de onda que toma en cuenta los aspectos dinamicos, para el cual tenemos simulaciones computacionales preliminares que permiten plantear hipótesis razonables y, en algunos casos, sugerir respuestas tentativas a diversas preguntas. Dichas hipótesis deberán ser objeto, en última instancia, de un estudio experimental detallado, y creemos que deben considerarse, más que otra cosa, como un punto de partida.

\section{Arqueoacústica}

La acústica arqueológica o lo que podriamos llamar arqueoacústica no es una disciplina nueva, en los últimos años se ha venido desarrollando desde diversos puntos de vista, al grado de que existen esfuerzos por sistematizar sus objetivos, contenido, métodos, etc. ${ }^{1} \mathrm{Al}$ menos uno de los ejes conceptuales plantea la posi-

'Véase, por ejemplo, I. Cross y A. Watson (2006), asi come varios articulas relacionados con esta misma referencia. 
bilidad de realizar mediciones acuisticas para obtener información sobre diversas actividades humanas, religiosas, politicas, sociales, etc., de diferentes culturas en el pasado y, en su caso, establecer también conexiones con las correspondientes manifestaciones en la actualidad. En el caso de las culturas mesoamericanas y de la cultura maya en particular, existe una gran variedad de posibles lineas de investigación en este sentido, algunas de las cuales se encuentran relacionadas con efectos acústicos específicos en diversos sitios arqueológicos, lo que discutiremos en la siguiente sección. Citamos como ejemplo el estudio realizado para tratar de correlacionar la estructura arquitectónica del Grupo Norte en Palenque con las representaciones escénicas y su significado social. ${ }^{2}$

Nuestro objetivo es delinear un marco básico para la investigación de algunos de los fenómenos acústicos, en particular en el caso de la cultura maya, que constituye el foco de nuestro interés. Queremos enfatizar que para establecer este marco, es necesario incorporar tanto aspectos cuantitativos, medición y caracterización de espacios, materiales constructivos e instrumentos musicales, es decir, elementos que forman parte de la ingenieria acústica, como herramientas conceptuales. Con respecto a esto ültimo nos referimos a que para formular hipótesis razonables y poder establecer un vinculo útil entre la teoría y la práctica, son indispensables los modelos matemáticos tanto en un plano abstracto como en el nivel de su implementación numérica. En los fenómenos acústicos ocurre algo similar al caso de la arqueoastronomia, donde es indispensable el bagaje matemático asociado con el estudio de los movimientos de los cuerpos celestes.

Dado que el fenómeno sonoro y auditivo debe entenderse en varios planos, es necesario tratar cada uno de éstos y sus relaciones. Nos referimos a los aspectos:

a) Físicos estrictamente: el sonido como un fenómeno natural.

b) Fisiológicos y cognitivos.

c) De significado, como parte de manifestaciones culturales, religiosas, artisticas, etcétera.

Hacemos hincapié en que éstos son únicamente algunos de los aspectos por considerar, y en que la lista no pretende ser exhaustiva.

Por otra parte, una vez delimitados estos aspectos, es posible ser más sistemático. En relación con el punto (a). el sonido como fenómeno natural, es necesario considerar un protocolo de medición acústica. Empezando por las fuentes que se van a usar para producir sonidos, por ejemplo, en el caso en que se busque caracterizar un eco, o el patrón de frecuencias aumentadas en algún recinto, o caracterizar acústicamente un instrumento musical..$^{3}$ Es también importante considerar los materiales involucrados, pues, sobre todo en el caso de edificios, las restauraciones y reconstrucciones pueden haberse hecho, como sucede en muchas ocasiones, con materiales diferentes a los originales, alterando las propiedades acuisticas de la construcción o recinto como un todo. Quizá

\footnotetext{
${ }^{2}$ F. Zallaquett Rock (2006)

${ }^{3}$ idem.
} 
de menor relevancia, pero no obstante importantes, son las alteraciones en las caracterizaciones acústicas debidas a cambios de temperatura, humedad u otras condiciones fisicas. Todo esto debe estar integrado en un sistema de medición, almacenamiento y procesamiento de datos.

La elaboración de modelos matemáticos sólo puede iniciarse de manera detallada una vez que los datos anteriores estén disponibles. Desde el punto de vista matematico, puede resumirse el estudio acustico de un sistema como el plantear y resolver una ecuación especifica, la llamada ecuación de onda (véase la sección 5). El poder resolver la ecuación de onda servirá no sólo para recrear virtualmente condiciones que seria imposible reproducir experimentalmente, por ejemplo, el suponer que un recinto estaba totalmente cubierto de cierto estuco con determinadas propiedades acústicas, o que alguna pared u otro elemento arquitectónico ahora faltante estaba presente en la conformación original, etc. sino también como herramienta predictiva y de verificación de hipótesis. Seremos más explícitos a este respecto más adelante.

En cuanto al punto (b), aspectos fisiológicos y cognitivos, hay que recordar que el fenómeno auditivo requiere un receptor e, inevitablemente, un procesamiento y decodificación de la señal sonora. En este sentido, la caracterización acústica de un sistema arqueológico debe contemplar la forma en que son percibidos por el ser humano las diversas manifestaciones acústicas. Es importante señalar que en este punto, es indispensable incluir consideraciones de carácter psicoacuistico. ${ }^{4}$ Cabe apuntar que uno de los puntos más delicados son las extrapolaciones directas de la forma en que percibimos el sonido en la actualidad con la forma correspondiente de un oido "maya". En otras palabras, cualidades como "fuerte" o "débil" deben ser consideradas en un contexto relativo. No sólo eso, y esto nos lleva al último punto, (c), el significado de los sonidos puede variar considerablemente dependiendo del contexto cultural especifico. Por mencionar un ejemplo sencillo, los sonidos y notas graves en la Grecia Clásica estaban asociados a objetos o movimientos pesados, y los más agudos, a objetos o movimientos ligeros. No fue sino hasta mucho después, quizá de manera natural por asociación con la notación musical occidental, que la correspondencia con alturas (grave-bajo, agudo-alto) fue establecida. Es en este último punto en donde resulta más dificil llegar a conclusiones definitivas, $y$ únicamente con la colaboración de especialistas en las diversas disciplinas involucradas se podrán dar resultados.

\section{Antecedentes de trabajos sobre la acústica prehispánica en la zona maya}

En relación con la acústica de instrumentos mayas prehispánicos, Velázquez (2002) examinó en su tesis de licenciatura, tres silbatos de barro dobles que se

\footnotetext{
${ }^{4}$ Nos referimes, por ejemplo, al caso de las curvas Fletcher Munson, por mencionar uno de los ajustes mâs conocidos (sobre este tema, véase F. Richard Moore [1990|t.
} 
encontraron enterrados en templos de la Pequeña Acrópolis (650-800 d. C.) de Yaxchilán. Estos silbatos fueron elaborados en forma de ranas en posición de canto [C-381 y C-466). El autor encontró que las ranas de barro podian sonar como las ranas naturales y que, posiblemente, sus coros se usaron en ceremonias del dios maya de la lluvia y el agua Chaac y en otros ritos chamánicos. Esas posibilidades se infirieron considerando que los mayas creian que los cantos de las ranas eran propicios para solicitar o atraer la lluvia, y se usaron en la ceremonia de Ch'a Ch'aac. Aun hoy, los maiceros celebran la ceremonia Ch"a Ch'aac, donde los niños tradicionalmente hacen los ruidos imitando el croar de ranas y sapos. Este estudio planteó la posibilidad de utilizar programas de computación para el anälisis de las caracteristicas acústicas de los silbatos.

Otra investigación importante fue realizada por Flores y Flores (1984: 439443) sobre 355 silbatos mayas. Incluyeron un análisis de tono-volumen interno de los silbatos, aunque utilizando las notaciones musicales de un piano para asociar el sonido generado por los silbatos, los cuales a veces generan tonos que no están clasificados a la usanza occidental.

En cuanto a las características acústicas de los espacios abiertos, Zalaquett (2006) estudió tanto los cuartos de los templos del Grupo Norte de Palenque, como la plaza, los instrumentos musicales y el estuco que recubria la estructura, y logró detectar que los cuartos más idóneos para interpretar instrumentos o también para cantar o dar discursos se encontraban en los Templos I y III, ya que su calidad auditiva es muy variada, y se comportan como una concha acústica, lo que quiere decir que aumentan la mayoria de las frecuencias, y por ende se escuchan más fuerte. Revisando las evidencias materiales, observó que frente al Templo I, en el escombro se encontraron varias figurillas y tres silbatos. Lo mismo sucede frente al Templo III, donde se ubicaron varios metates de ofrenda y algunos silbatos. Como en la base de este templo se encuentra una amplia terraza, pudo inferir que era un lugar apropiado para efectuar presentaciones musicales y llamados a la población que se escucharan en un rango espacial más extendido.

Este descubrimiento de las cualidades acústicas de algunos cuartos permite interpretar las posibles actividades que se realizaban en los cuartos donde muchas veces se encuentra una escasa evidencia material. Esto demuestra que los mayas construyeron distintos cuartos con diferentes dimensiones y materiales. utilizando un conocimiento sobre el sonido, lo que permitia la transmisión de mensajes y música a una mayor distancia y público, logrando involucrar a una mayor cantidad de población en una actividad realizada en el interior de éstos.

En cuanto a los análisis en la plaza, obtuvo áreas idóneas donde se pueden ubicar mejor los músicos y los espectadores. Ubicando a los músicos sobre los puntos 4,6 y 7 , o sea frente a los Templos V, IV y III, se alcanza una gran extensión auditiva. Desde estos puntos, los músicos pudieron acompañar ritos que irian en conjunto o independiente del Templo del Conde, dirigiéndose a una gran población localizada en la plaza. 


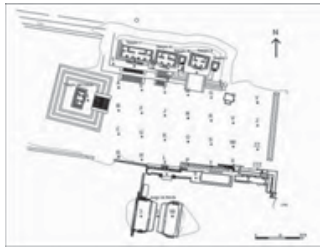

Mave 1. Thussectos y pukios donde se efectuaron las mediciones acústicas en el Grupo Norte de Palenque (tomado de Zalaquett, 2006: 188)

\section{Manifestaciones escénicas en la zona maya}

Los antiguos mayas y otros grupos mesoamericanos mostraron gran interés en invocar a los sentidos, especialmente el oido, ta vista y el olfato. El medio principal para la comunicación sinestésica (la liberación de una sensación a través de otra, o en lenguaje técnico, una modalidad de experiencia cruzada, la percepción de sonidos por ejemplo, da la sensación de colores) era la escritura. En el arte del Clásico maya y en su escritura, habia una distinción entre el sonido y las canciones de belleza y alabanza. Una concha tallada del Clásico Tardio muestra a un músico tocando un par de sonajas mientras está cantando; el sonido delineado por una larga virgula sale de su boca. La virgula de la palabra es un signo distintivo que consiste en una cabeza joven, con la boca abierta, de la cual emana una virgula que termina en una flor. Contextualmente, como en Bonampak, estas funciones del signo de la palabra son un título que acomparia a los músicos, generalmente los que tocan las sonajas. Una clave para su significado puede ser encontrada en su signo sufijo, ma, y en una forma completamente fonética del periodo Clásico Temprano que al parecer describe al dueño de una trompeta de concha: ambas palabras indican que su lectura es k'ayoni:m-ma o k'a-yo-ma "cantante" (Houston y Taube, 2000: 261-294). 
Las virgulas de palabras son relativamente comunes en el arte del Clásico maya. En una imagen (figura 1) ambientada en un Juego de pelota, el artista muestra efectos de ecos en los lugares arquitectónicos a través de la extensión de virgulas que salen de los labios humanos. Las implicaciones analiticas del ènfasis puesto en los espacios abiertos indican que los arqueólogos deben poner más atención a la acústica de los edificios. Por ejemplo, muchos visitantes comentan sobre los efectos de susurros o las impresionantes distancias que el sonido puede viajar en las plazas, en la parte superior o en la base de las escaleras. Es improbable que los mayas no se hayan percatado de estas cualidades, y que, como los diseñadores y constructores, no utilizaran el juego entre el sonido y la palabra con diversos propósitos. Esos espacios no estaban aislados sino que estaban intermitentemente llenos de retórica y canciones (Houston y Taube, 2000).

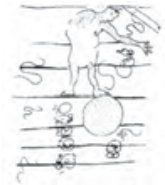

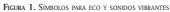
(tomada de Houston y Taabe, 2000: 280]

El variado espectro auditivo de los mayas también se puede estudiar en los diccionarios realizados por los españoles, como por ejemplo, en el caso de los tzeltales de Copanaguastla, donde se describen sonidos vinculados con las estridencias, roces, graznidos y murmullos de animales, maderas, piedras o cosas que se rozan o quiebran, o de las que se extraen sonidos musicales fincluso diferenciando éstos según el utensilio con que se les arranca el sonido y clasificando además la suavidad, estridencia e incluso la "soberbia" del sonido obtenido). En cuanto a los sonidos que desprende el cuerpo humano, aparecen en multiples entradas. Se especifica el ruido que hace la gente que pasa, se emplean voces que dan cuenta desde el rumor sordo comparable al que provocan el fluir del agua o el correr del viento (tinitet), hasta el transcurrir del gentio sobre la tierra, el murmulto "descomedido" que provoca una multitud (cunan: juntarse, amontonarse) 
que hormiguea (tictonet). bulle (nic) y se atropella (Ruz, 1995: 46). En el caso de los animales, las aves, por ejemplo, cuyos gorgeos, cantos o graznidos era importante conocer, ya que sabemos que se acostumbraba imitarlas para atraerlas hacia el cazador (en busca de sus carnes o sus plumas).

Asi como existian estas especificaciones de los sonidos, los mayas también fabricaron variados instrumentos musicales, los que han sido clasificados en idiófonos, aerófonos y membranófonos. Dentro de los primeros se incluyen las campanas de cerảmica, los raspadores (de hueso, piedra, etc.), sonajas de múltiples tipos, que albergaban semillas o piedras, conchas de tortuga, calabazos, bastones y tunkules (en sus origenes propiamente un antecesor más que un equivalente del teponaxtli nahua.) Los aerófonos están conformados por silbatos, ocarinas (o flautas globulares), caracoles marinos (con el vértice cortado a manera de boquilla), flautas y trompetas de madera (sacabuches) (Ruz, 1995). Como representantes de los instrumentos con membranas estarian los tambores de marco (como los que se observan en tres placas de Lubaantún, del Clásico Tardio), cerámica (de olla o timbales, que aparecen en el Codex Tro-cortesianus o Codice Madrid y la pintura mural de Uaxactun) o de madera (tal el equivalente de Huehuetl grande de los nahuas, llamado zacot'na entre los yucatecos).

Era de tal importancia la adecuada ejecución de un instrumento musical, que en una breve pero significativa nota de fray Alonso de Ciudad Real acerca de los chol-lacandones, hacia 1585 , se ilustran los problemas que podia conllevar una ejecución desafortunada. Cuando hablan sobre una ceremonia sacrificial apunta:

Legando el día en que habia de morir (el indio prisionero) le sacaron de la cárcel. y llevado al baile o mitote, comenzaron su fiesta, quiso su ventura u ordenolo asi Dios, que el que estabar taitendo el teponastle, que es un instrumento de madera que se poye meda legua y más, erró al golpear y el compás de la música, y teniendo esto por agiiero y mala seña, el sacerdote de los indios mandó que no pasase la fiesta delante ni se hiciese por entonces el sacrificio y que muriese el tañedor que habia hecho aquella falta, tan grande a su parecer [...] [Ruz, 1995).

Los instrumentos son además elementos que permiten integrar a las nuevas generaciones en los ritos y hacerlos participes de estas actividades, con lo que se pretende asegurar la continuidad de éstos en el tiempo, estableciendo lazos con los niños y las actividades de los adultos. En Calkini, son entregados a los niños pequeños silbatos con efigies antropomorfas y zoomorfas y flautas para usarios durante las ceremonias del Ch'a Ch'ooc y Semana Santa para pedir la lluvia, asi como sucede también en la ceremonia del Día de los Muertos en la misma localidad, en Campeche y en Ticul, Yucatán.

Para estudiar las representaciones mayas, es importante concebirlas en su totalidad, donde el mito pasa a ser una descripción de los acontecimientos, y el baile, una demostración visual de ellos. Las danzas rituales, las procesiones simbólicas, las comedias, los juegos o representaciones teatrales al aire libre son, 
desde tiempos inmemoriales, parte integrante del culto en la zona maya. Perpetuaan escenas miticas y exaltan los principios morales, normativos de la conducta humana, poniéndolos, en forma alegórica, al alcance del público (Muñoz, 2000: 41).

En la danza maya no se intenta copiar a la naturaleza, sino revelar simbólicamente su esencia mágica y sobrenatural, formando una identidad de grupo al ser ejecutadas y transmitidas en público. Se produce asi una interacción social que logra formar una unidad coherente y participativa para los asistentes y los ejecutantes. Se danza para estar junto a los ancestros, para comunicarse con las deidades. En estas reuniones participan tanto los ejecutantes como los observadores, que entregan ofrendas y algunas veces danzan, aplauden o cantan junto a la música. Con ello se crea un momento en el que todos participan en común con un objetivo y en un lugar, lo que permite que la comunicación en estas actividades sea más fluida, sin diferencias que se puedan presentar en actividades cotidianas. En estos momentos es cuando existe una identidad de grupo nueva, con la que todos se sienten cercanos y de la cual todos participan.

En cuanto a su temática, existen representaciones públicas descritas en los vocabularios realizados en la zona maya, por ejemplo, en el Diccionario de Motul (Arzápalo, 1995: 1319), elaborado cerca de 1577, se registran nueve nombres de obras teatrales que parecen referirse a representaciones de carácter cómico, farsas donde la gracia estaba en el decir, y que muchas veces servian para expresar criticas sociales. Entre ellas se encuentran:

a) Ah Canche Caan o El Escabel del Cielo y que dice ser cierto entremés (pieza breve, cómica) que hacen los indios.

b) Ah Con Cutz o sea El Vendedor de Pavos Silvestres.

c) Ah Com Cum o sea El Vendedor de Ollas.

d) Ah Con Ic o sea El que vende Chiles.

e) Ah Con Tzatzam o El que vende Enredos.

f) Ah Cuch Uitz o sea El que carga o gobierna la Sierra Alta.

g) Ah Socchiil Mo o sea La Guacamaya de la Boca Blanca o De los negocios falsos.

h) Ah Soc Hool Paal o sea El Chico de la Cabeza Blanca.

i) Ah Pakal Cacau o sea El Cultivador de Cacao (Barrera Vásquez, 1965: 11).

Los ejecutantes de estas obras tenian sus especializaciones, las cuales están detalladas en los diccionarios yucatecos (Muñoz, 2000: 33), donde se describen los nombres que recibian los actores, danzantes, ilusionistas, ventrilocuos, cantantes y directores de las diferentes disciplinas. Como algunos ejemplos, tenemos a:

- Ah Balzamoob: juglares o actores de representaciones teatrales.

- Ah Cuch Tzublal: director de arte escénico, principal que tiene cuidado de los danzantes o farsantes que se juntan en su casa y se impone.

- Ah Xinib Che': el que anda en zancos largos de madera. 
- Ah Okot: danzante.

- Ah Kayom: cantor.

- Ah Noh Cal: el gran garganta, el gran voz, el de la voz recta.

- Ah Süan Can o Ah Sïan Than el que sabe muchas historias. Especie de dramaturgo.

- Ah Taah: decidor.

- Ah Tuz Kay: compositor de cantos.

- Bacab: representante, juglar.

- Balt's amyahr representar.

- Ezyah: el que hace ilusionismos.

- Holpop: maestro de canto, que entona y enseña lo que se ha de cantar.

- Tah Ach: representante cómico, actor de teatro.

- Ta'ah: farsa, representación

Estos especialistas tenian un lugar determinado de reunión para estudiar y ensayar sus bailes, llamado Popolna, cuyo encargado era el Holpop, quien guardaba alli los instrumentos y el atuendo. Esta casa no era sólo para eso, sino también para tratar cosas de la comunidad (Barrera Vásquez, 1965: 11). Como se puede determinar, el teatro y la danza eran artes comunales y también eran una institución permanente de la comunidad.

Gracias a los diccionarios en lengua tzeltal sabemos que también en el poblado de Copanaguastla, Chiapas, se acostumbraban las escuelas de danza: sus nombres eran caynob acot y nopob acot. El primer término está vinculado con la voz para baile (acot) y relacionado al parecer con cayn, imitar a otro, y cayogh, canto, cayom músico y el tambor con cuero. Lamado cayb. Nopob, por su parte, se traduce de dos maneras: "comparar, concordar, como las voces" y "ordenamiento", lo que permitiria traducir nopob acot como el lugar donde se hacia "el ordenamiento del baile", sin descartar, dada su primera acepción, que estas escuelas "de danzar" hubiesen fungido tambièn como escuelas de canto (Ruz, 1995).

Como se supone por los textos, los danzantes y músicos tenian un lugar estable dentro de la sociedad maya en Yucatán y Chiapas, asi como también una casa donde se reunian.

En cuanto a los bailes en particular y las descripciones realizadas por los frailes que los observaron, eran presentados en ocasión de las grandes fiestas, dedicados a los dioses, eran de carácter dramático y pantomimico. Se trataba más bien de espectáculos danzantes que de simples representaciones de baile, los cuales evocaban temas mitológicos y en parte temas históricos; servian también para glorificar las luchas victoriosas contra pueblos enemigos. En la lengua cakchiquel se les llama nuik'um tzij" (guirnaldas de palabras), aludiendo al sentido poético atribuido a los parlamentos o recitados; además de la declamación de textos, complementados por gestos pantomimicos, está la comedia improvisada, como el baile de los Gracejos y de varias plumas y de muchos sartales de chalchiguijs (Vela, 1972: 517). 
Otro documento bastante completo es el Thesaurus de la lengua Cakchiguel, realizado por fray Tomás de Coto, que describe algunos bailes (Xahoh) que se realizaban: en un baile voltean un palo redondo con los pies (vingh), en otro tocan unas varas largas y huecas (lotz tun), que suelen hacer entre dos o tres con sus mascarillas, a modo del matachín, y se meten unos palos y huesos por la boca y garganta, y por las narices, dándose golpes en los pechos ( $\mathrm{Xq}$, ull). A los que bailan llaman: he $x q, u l$, he logom (Coto, 1983: 61). En el mismo texto se traduce al canto como bix, y a los musicos como bixanel, ah bix (Coto, 1983: 364).

\section{Fenómenos acústicos mayas}

En nuestro intento por hacer una búsqueda exhaustiva de la investigación sobre efectos acústicos en sitios mayas, nos sorprendió, al platicar con varios arqueólogos, la gran cantidad de anécdotas y experiencias personales relacionadas. Por ejemplo, el doctor Ernesto Vargas, del Instituto de Investigaciones Antropológicas de la UNAM, nos comentó que el conocido efecto del juego de pelota de Chichén Itzá era utilizado por Sylvanus G. Morley para impresionar a sus invitados durante las comidas que organizaba en ese lugar. Parece que Morley colocaba la mesa y las sillas en donde estaria toda la gente en un extremo de la cancha, mientras que dejaba otra mesa con un fonógrafo en el otro extremo. Por supuesto que los asistentes preguntaban al arqueólogo por qué dejaba el aparato tan lejos y todos quedaban impresionados de que la música se oyera "como si el fonógrafo estuviera junto" cuando Morley ponia un disco. El mismo efecto al aplaudir en uno de los extremos y el eco producido es explotado por todos los guias durante los recorridos turísticos. También en Chichén ltzà se registran otros dos más en la pirámide de El Castillo, asi como en la escalinata. Si una persona habla en voz baja en la parte superior de la pirámide, es escuchada sin problemas por otra en la base de las escaleras. También se escucha un efecto curioso para alguien en la base de la escalera principal, pues los pasos de las personas que van llegando a la parte superior se perciben como "gotas de lluvia".

Como éste, hay una infinidad de otros efectos que mencionamos, aunque sea para demostrar que una recopilación y clasificación es muy necesaria y que de hecho, forma parte de nuestro proyecto de trabajo actual. ${ }^{5}$ En diversos sitios (en particular Chichén Itzá) se hace mención de "piedras musicales" o "conos musicales" que consisten en una serie de piedras colocadas juntas, que al ser golpeadas producen diversos tonos. Señalamos también que algunos de estos fenómenos no se limitan a sitios mayas, sino que están presentes en otros asentamientos

\footnotetext{
${ }^{5}$ F. . Elizondo Garza [1996) en "Turismo acuistico en Chichén Itzà", ponencia presentada en el Zer. Congreso Mexicano de Acvistica, pone ya de manifiesto la necesidad de estudiar, analizar, documentar e irwestigar efectos acüsticos en Chichein Itzá. El documento está disponible en http: Yrwww.gama. fime.uanl.mx/acustica/articulovchichen.doc iconsulta hecha el $7 / 12 / 2007$ ). También, más recientemen: te, Wayne Van Kirk 12002$\}$.
} 
prehispánicos, como Monte Albán y Teotihuacan. Este último podria estar relacionado con el barrido acústico de la cola del quetzal en Chichén Itzä, y ha sido también estudiado por S. Beristáin, C. Coss, G. Aquino y J. Negrete (2002) y por J. Carrera y S. Beristäin (2002).

En Xel-Ha se reporta que las conversaciones mantenidas en un extremo de una cueva pueden ser oidas sin problema a varias decenas de metros de distancia.

También en Tulum se reporta que arqueólogos trabajando en lugares especificos del sitio podian, sin tener que levantar la voz, hacerse oir por otros trabajadores a distancia considerable. Es en este mismo sitio en donde, de acuerdo con la tradición, se da un efecto acústico notable que merece un estudio concienzudo. En el techo de uno de los edificios están colocados un cilindro junto a un anillo, ambos de piedra, y el primero en el mismo plano que el segundo. Algunos de los guias locales aseguran que cuando los vientos alcanzan velocidades altas, por ejemplo durante el acercamiento de un huracán a la costa, es posible oir el silbido producido por el anillo a varios cientos de metros. De ser cierto, este dispositivo constituiria una de las primeras "alarmas meteorológicas", pues hay que recordar que Tulum es una ciudad amurallada, y sus murallas podrian haber servido de protección a la población. Hasta dónde se puedan validar estas conjeturas, que en primera instancia resultan más que sorprendentes, depende de la integración de los elementos metodológicos que mencionamos anteriormente. En particular, con relación a este último efecto, el mecanismo hidrodinámico y acustico responsable de producir un silbido podría deberse a la interacción de una estela vorticosa producida por el cilindro, entrando en resonancia con el anillo. Si éste es el caso, la comprensión fisica y matemática del fenómeno dista de ser trivial. ${ }^{7}$ No sólo eso, sino que seria deseable sustentar estas hipótesis en datos arqueológicos y experimentos.

En diversos sitios mayas, de los cuales Palenque es quizá el más conocido (hemos ya mencionado Tulum), se observa la amplificación de la voz en puntos especificos. Incluso Morley menciona ya la necesidad de estudiar este efecto y sustentar la afirmación de que muy bien pudo ser utilizado por los sacerdotes y gobernantes para dirigirse a un grupo numeroso de personas reunidas en ceremonias públicas. Este fenómeno $\mathrm{y}$, en general, el profundizar sobre el diseño acústico de las plazas y otros espacios arquitectónicos y su posible uso por parte de los constructores en ritos, ceremonias y otro tipo de manifestaciones escénicas, claramente merece atención detallada.

Para finalizar esta lista informal de efectos acústicos en sitios arqueológicos, nos parece significativo mencionar lo que ocurre en la Plaza del Arroyo en Tajin. Al aplaudir enfrente de cada uno de los cuatro edificios que limitan la plaza, el eco producido parece surgir de atrás del edificio correspondiente y re-

\footnotetext{
${ }^{6}$ Comanicación personal del doctor Ernesto Vargas.

T Este mismo grupo de trabajo ha plasteado ya la posibailidad de realizar experimentos en el tuinel de viento de la Torre de Ingenieria de la UNAM con el apoyo del doctor Neftali Rodriguez y de desarrollar algunos modelos especificos.
} 
gresar por los costados. En cualquier caso, seria deseable hacer mediciones más precisas. De ser éste el caso, los modelos matemáticos que se han propuesto hasta ahora (fundamentalmente modelos en dos dimensiones espaciales), serian inadecuados y haria falta incorporar la geometria real, es decir tridimensional, del complejo arquitectónico. No deja de ser sugerente el hecho de que el sitio del Tajín tiene una asociación con el relámpago y el trueno. Quizá pueda interpretarse el eco que surge de atrás de las piramides como una manifestación de este fenómeno natural que muy bien pudo haber sido incorporada en algún ritual o ceremonia.

Por último, complementando a los estudios acústicos orientados al ámbito arquitectónico, se encuentran aquellos que es necesario realizar para caracterizar las propiedades de los instrumentos musicales. Además del interés intrinseco que esta investigación tiene, las fuentes sonoras en el contexto de ceremonias y otras manifestaciones escénicas eran, de forma natural, los instrumentos musicales, de los cuales existe todavia una variedad suficiente para poder emprender este trabajo. En el caso maya, hasta donde nosotros sabemos, no existe el equivalente de la valiosísima obra de R. Stevenson (1968) sobre la música azteca e inca al tiempo de la conquista.

\section{La Cola del Quetzal en la pirámide El Castillo en Chichén Itzá}

En esta última sección describimos en más detalle el efecto del barrido acústico (chirp en inglés) producido al aplaudir frente a la escalinata principal de la pirámide de El Castillo en Chichén Itzá (figura 2). Asimismo, resumimos lo que constituye, hasta donde hemos podido corroborar, las investigaciones más recientes al respecto. El efecto se produce, como ya mencionamos, cuando una persona colocada en el mismo plano de la escalinata aplaude. El eco producido ha sido denominado "la cola del quetzal", en principio por su semejanza con el sonido emitido por este animal. ${ }^{8}$ Cabe mencionar que el quetzal era un ave muy venerada por los mayas de Yucatán a pesar de no ser originaria de la región sino de las selvas de Chiapas. Es daro que el efecto se produce como resultado de la interacción del campo acústico generado por el aplauso con la estructura periódica de las escaleras. Lubman propone que el fenómeno se debe esencialmente a la difracción de Bragg ${ }^{9}$ y procede a reproducir el sonograma ${ }^{10}$ construido con base en la grabación del barrido. ${ }^{11}$

\footnotetext{
'Para comparar estos dos sonidos puede consultarse las grabaciones proporcionadas por D. Labman en su sitio: httpolwww.acasa.orgMayanPyramid.htm (consultado el $7 / 1 / 2007)$.

' Véase, por ejemplo, http:/hww.esere.stonybronk.edu/Projectjawa/Bragg' (consultado el g/1/ 20071

"日 sonograma de un registro sonoro es una representación gräifica de las frecuencias cque componen dichn registro come función del tiempo.

ir El sonograma está tomado de httpothwww.ocasa.org/MayanPyramid2.htm fconsultado el 8/12007). D. Labman presentó además sus desarrollos en diversas pláticas, reportadas en: D. Lub man $11998,1999 a, 1999 b, 2002$ ).
} 


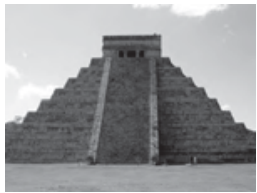

Fouk 2. El. Custuo, Chenen 1TzA

La difracción de Bragg se descubrió a principios del siglo $\mathrm{xx}$ al analizar los patrones de difracción que se forman con rayos $\mathrm{X}$ sobre estructuras cristalinas (figura 3).

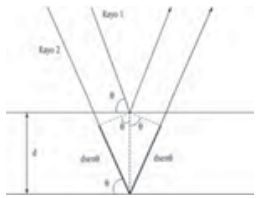

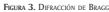

Los rayos $\mathrm{X}$ pueden pensarse como ondas senoidales (figura 4). Cuando dos ondas con la misma longitud de onda interactúan, el resultado depende de la di- 
ferencia de fase, es decir, con qué parte de una onda coincide la cresta de la otra. Si las crestas coinciden, las ondas se suman (interferencia constructiva). Si la cresta de una coincide con el valle de la otra, se cancelan (interferencia destructiva). Entonces, cuando llegan los rayos $\mathrm{X}$ a una estructura cristalina, los que se reflejan en una capa interactuan con los que se reflejan en la siguiente y, dependiendo del ángulo de incidencia, la frecuencia de los rayos y la separación entre las capas atómicas, se tiene interferencia constructiva o destructiva.

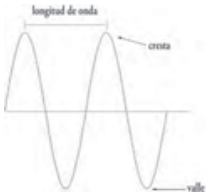

Ficura 4. Onem sevoinal

Volviendo al ămbito acústico, un aplauso puede pensarse como una suma de ondas senoidales con diferente longitud de onda $\lambda$ y correspondiente frecuencia $\mathrm{f}=\mathrm{v} / \mathrm{h}$, siendo $\mathrm{v}$ la velocidad de la onda. Por la forma de los escalones, algunas frecuencias sufren interferencia constructiva y otras destructiva (véase la figura 5), y en el eco, de acuerdo con la explicación de Lubman, sólo se escuchan las primeras.
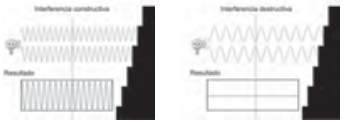

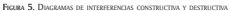


Lubman argumenta además que las dimensiones de los escalones favorecen el reforzamiento de frecuencias en el rango de las correspondientes emitidas por el quetzal, y explica cualitativamente el hecho de que las frecuencias más bajas sean las que sufren un mayor retraso, tal como se muestra en el sonograma (figura 6). Posteriormente también justifica estos resultados cuantitativamente mediante el uso de un programa de cómputo (Mathcad) para reproducir las curvas que aparecen en el sonograma anterior. Sin embargo, la forma en que la simulación se llevó a cabo no se presenta. La conclusión de Lubman en relación con la intencionalidad en el diseño maya para aprovechar este efecto, es que no se trata de una coincidencia. Por otra parte, el mismo Lubman en su sitio de Internet menciona algunas criticas hechas a su trabajo por arqueólogos. En resumen, puede decirse que dichas criticas se refieren a la falta de sustento histórico y documental de sus conclusiones, pero tampoco proveen un argumento definitivo que permita descartar la intencionalidad en el diseño. Desde el punto de vista fisico y matemático, el estudio de Lubman es retomado por Declercq et al (2004). En su trabajo mencionan que "el efecto (del barrido del quetzal) ha sido objeto de numerosas pláticas dadas por Lubman y otros. Lubman ha enfatizado el hecho de que el barrido del quetzal se debe a la difracción de Bragg. Sin embargo, no se ha presentado una simulación como tal de dicho efecto, excepto por algunas

\section{Handelap belowe W Starcase of Pyearid of Kukulsan 9/20198}

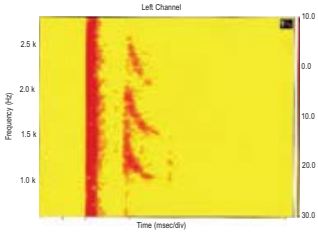

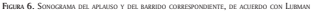


simulaciones heuristicas basadas en teoria de rayos o un enfoque heuristico para el caso en el que el ángulo de incidencia es de $45^{\circ}$ medidos en relación con la normal a la superficie". ${ }^{12}$ En el trabajo de Declercq et al., hay también una discusión sobre el significado del quetzal en la cultura maya e incluso se menciona que un eco en dicho contexto cultural puede representar un espiritu (no se da referencia de esta última afirmación). Por eso, argumentan los autores, la generación de un eco imitando al quetzal puede no tratarse de una coincidencia. Antes de entrar en más tecnicismos sobre este trabajo, diremos que, de forma general, se concluye que efectos similares ocurren en otros sitios (Sri Lanka y otros sitios arqueológicos en México, por ejemplo Uxmal, figuras 7 y 8) y los autores no presentan ninguna opinión definitiva acerca de la intencionalidad.
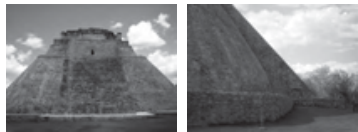

Ficurus 7 Y 8. Pä́mbe del Aonno, Uxual.

Sin embargo, es importante hacer notar que el hecho de que efectos similares aparezcan en otros sitios en Mesoamérica, en particular en Teotihuacan, en donde el quetzal ocupa un lugar fundamental en la simbologia, da pie a estudiar si existe una relación explicita entre los edificios en donde efectivamente aparece este efecto y su función religiosa. Si es posible entender en detalle los elementos fundamentales que deben estar presentes en una construcción para que se produzca el barrido del quetzal, y si dichos elementos se encuentran en diversos edificios de diferentes sitios, entonces habría un elemento objetivo mucho más concreto para apoyar o descartar la hipótesis de que el efecto fue incorporado propositivamente por los constructores. Apuntamos también que en el trabajo de Declercq et al. se presenta una explicación convincente del efecto de "gotas de lluvia" en la pirámide de El Castillo y cabría la posibilidad de que el mismo mecanismo fuera responsable del efecto de amplificación de la voz desde la parte alta de la pirámide. Otros dos aspectos relevantes de este trabajo son los siguientes. Primero, se hace énfasis en que la naturaleza del eco puede depender fuertemente de la

${ }^{12}$ La cita está tomada de la introducción de la referencia anterior (nota 24). La traducción es de Jas autores, asi conso los paréntesis rectangulares. 
naturaleza del objeto que sirve de fuente, y por ello se hace ver la necesidad de realizar experimentos con diferentes instrumentos, como tambores o madera percutida. Segundo, si bien se concluye que el eco producido por la pirámide consiste en el sonido difractado por la escalera y que este proceso está relacionado con la difracción de Bragg, se menciona que otros efectos son relevantes, algunos de los cuales están relacionados con la propagación del sonido sobre las escaleras mismas. Por lo anterior y dadas otras consideraciones que expondremos más adelante, también es posible concluir que no se cuenta con una descripción temporal precisa del eco (es decir, como se oye en tiempo real), asi como de los mecanismos precisos que lo causan, qué tanto influye la geometria de la pirámide y cuál es con precisión la influencia de las caracteristicas de los materiales que la forman o que la recubrieron. Retomando el objetivo de nuestro trabajo, si es que se quiere correlacionar el fenómeno acústico con elementos arqueológicos, arquitectónicos, culturales, etc. es indispensable tener una manera práctica de ver como inciden estos factores en la producción del eco.

En lo que resta del trabajo, describimos entonces la metodologia que proponemos, asi como algunas simulaciones preliminares.

El problema fundamental de la acústica, esto es, el de conocer el campo sonoro (distribución de presiones) como función de la posición y del tiempo, puede plantearse en términos matemáticos como el problema de resolver la ecuación de onda para dicho campo. ${ }^{13}$ Esta ecuación es

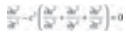

que representa esencialmente la segunda ley de Newton aplicada a una propagación en un medio elástico. En dicha ecuación, $u[x, y, z, t)$ representa la presión en el punto $(x, y, z)$ al tiempo t y c es la velocidad de propagación del sonido en el aire, que está determinada por las condiciones de temperatura, presión, etcétera, del medio.

Además de la ecuación misma, es necesario especificar las llamadas condiciones iniciales fen el caso que nos interesa corresponderian a la forma determinada por el aplauso, pulso o cualquier otra fuente que se utilice para producir la perturbación inicial), asi como las condiciones de frontera, que en nuestro caso juegan un papel fundamental, pues en ellas se incorporan las caracteristicas acuisticas (reflexión, absorción) del medio.

En la simulación que presentamos, se consideran las condiciones de frontera más simples, conocidas como condiciones de Neumann, y que corresponden a paredes ideales completamente reflejantes. Esto lo hacemos con el objeto de hacer ver que aun en este caso simplificado, se tienen ya una serie de preguntas e hipótesis que son dificiles de responder con un modelo de rayos estrictamente geométrico.

\footnotetext{
Déase, por ejemplo, LE Kinsler y A. R. Frey (1950).
} 
En la secuencia que sigue, aparece del lado izquierdo una escalinata, que representa la mitad de la piramide (en blanco); en azul se representa el espacio abierto circundante. A continuación detallamos los aspectos que nos parecen más relevantes al pie de cada una de las figuras, aprovechando para plantear aspectos experimentales por realizar, asi como preguntas abiertas e hipótesis tentativas. Por otra parte, observamos que estas simulaciones requieren, para que puedan ser útiles y permitir comparaciones con el experimento, hacerse de forma más realista. Para ello es necesario incorporar la geometría de la pirámide más exactamente y hacer mediciones precisas de la velocidad del sonido en el sitio, pues ésta es uno de los parámetros más relevantes en la ecuación de onda. Las condiciones de frontera deben adecuarse a los materiales usados, y para ello es necesario caracterizarlos acústicamente. Esto permitirá una comparación confiable para saber como pudo haberse oido el barrido en las circunstancias previstas por los constructores.

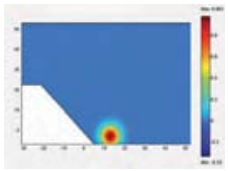

Figura 9

Inicio del aplauso (figura 9), que en este caso se simuló como un pulso gaussiano; corresponde al circulo con centro rojo oscuro. Las unidades están en metros, por lo que el observador se encuentra alrededor de $15 \mathrm{~m}$ del borde de la escalera. Hacemos notar que Declercq et al. consideran no sólo un pulso gaussiano, sino también una aproximación a un aplauso real. Sin embargo, dadas las limitaciones computacionales, tienen que restringirse a un numero menor de frecuencias (modos de Fourier), por lo que uno de los primeros aspectos por tratar es el de estudiar el efecto de la fuente, pudiendo generar datos iniciales que correspondan a fuentes realistas. De hecho, es también interesante mencionar, desde el punto de vista arqueológico, que no sabemos de ninguna referencia explicita sobre el papel que pudieron haber jugado los aplausos durante las ceremonias o ritos. 


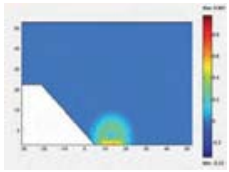

Ficater 10

El pulso comienza a expandirse (figura 10). Recordamos que Dedercq et al., en la referencia ya mencionada, señalan que el efecto del piso no es relevante para propósitos de la determinación del campo acústico en el nivel del observador. Si bien esto es cierto en lo que atañe a la selección de frecuencias, la imagen parece sugerir que es importante considerar dicho efecto en la evolución temporal. De hecho, uno de los aspectos que no se especifica de forma muy precisa en los trabajos tanto de Lubman como de Dedercq et al., es la duración aproximada del barrido. Si uno compara los sonogramas presentados por Declercq, pareceria que, de acuerdo con sus resultados, el eco calculado es más corto que el medido experimentalmente, aunque esta última afirmación requiere ser precisada, pues el nivel de ruido es grande. Ésta es otra consideración que nos parece importante, pues es quizá recomendable efectuar alguin proceso de filtrado al sonograma del barrido real.

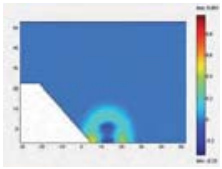

Ficusa 11 
En esta imagen (figura 11) se aprecia el pulso justo antes de que empiece a interactuar con la estructura periódica de la escalera. Nótese que todavia la propagación de la perturbación es simétrica.

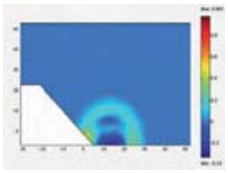

\section{Ficisa 12}

Empieza a haber interacción entre el pulso y la escalinata, que es responsable de la asimetria observada (figura 12).

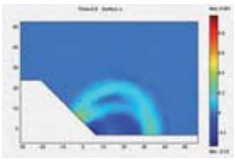

Ficusa 13

Hay un hecho notable que recalcar en esta imagen (figura 13): es clara la aparición de una estructura (arco más bajo y difuso de los tres) que proviene de la escalinata y que puede interpretarse ya como un eco. Por otra parte, el tiempo en el que esto ocurre, $0.9 \mathrm{seg}$. en la simulación, es consistente con este experimento. 


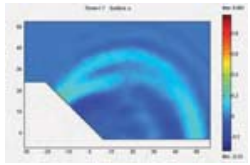

Ficane 14

El posible eco continúa propagándose y la perturbación inicial está por llegar al final de la escalinata (figura 14).

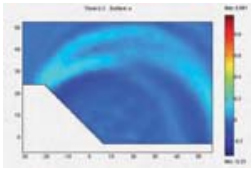

Ficaler 15

E pulso llega finalmente a la parte más alta de la escalera (figura 15). De las últimas cuatro imágenes parece también claro que la escalinata sirve como una especie de guía de ondas, lo cual parece consistente con el fenómeno discutido anteriormente: una persona hablando en la parte superior de la escalera es oida sin dificultad por alguien en la parte inferior. Esto sugiere por supuesto hacer la simulación correspondiente con la fuente acústica en la parte superior, asi como investigar si efectivamente el fenómeno descrito corresponde a lo que se conoce en fisica como una guia de ondas. 


\section{Conclusiones}

Una vez expuesto el contenido más técnico en relación con lo que pensamos que es un ejemplo representativo, nos parece importante retomar algunas consideraciones de carácter general.

Hemos mencionado varios ejemplos de efectos acústicos que se escuchan en sitios arqueológicos, en particular los sitios mayas, y recordado algunos de los estudios que se han hecho al respecto. Se dieron varios ejemplos que muestran que los mayas daban mucha importancia a la acústica. La conclusión básica es que hay mucho por hacer y que el estudio debe hacerse desde diferentes puntos de vista tanto cientificos como humanisticos. En la parte cientifica es indispensable adoptar diversos enfoques: analitico, asintótico y numérico, además, por supuesto, del experimental, pues ninguno de ellos en forma aislada puede proporcionar una comprensión completa de un fenómeno. La simulación presentada, junto con la discusión precedente, sugiere una serie de experimentos por realizar, asi como diversas preguntas e hipotesis que habria que contrastar y validar o refutar a la luz de un análisis arqueológico. El panorama es, en nuestra opinión, el de un campo extraordinariamente rico e interesante, en el que la inmensa mayoría del trabajo está por hacerse.

\section{BIBL.IOGRAFIA}

Arzápalo Marin, Ramón

1995 Diccionario Colepino de Motaf. México: UNAM.

Barrera Vasquez, Alfredo

1965 El libo de los cantares de Dzitbalché. México: INAH.

Beristáin, Sergio, Cecilia Coss, Gabriela Aquino y José Negrete

2002 "Tonal Response on the Stairway of the Main Pyramid at La Ciudadela, Teotihuacan Archelogical Site", f. Acoust. Soc. Ant., 112 (5). 2285 (articulo 3aAA4 en Proceddings of the first Pant-Americoniloerian Meeting an Acoustics).

Carrera, Jorge, y Sergio Beristáin

2002 "Theoretical Interpretation of a Case 5tudy: Acoustic Resonance in an Archaeological Site", J. Acoust. Soc. Am., 112 (5), 2285.

Coto, Thomás, fray

1983 Thesourus Verborunt. México: UNAM.

Cross, lan, y Aaron Warson

2006 "Acoustics and the Human Experience of Socially-Organized Sound". Archaeoocoustics, Chris Scarre y Graeme lawson (eds.). Cambridge, RU (McDonald lnstitute Monographs). 
Declercq, Nico F., Joris Degrieck, Rudy Briers y Oswald Lerny

2004 "A Theoretical Study of Special Acoustic Effects Caused by the Staircase of the E Castillo Pyramid at the Maya Ruins of Chichen-Itza in Mexico". J. Acoust. Soc. Am., 116 (6), 3328-3335.

Elizondo Garza, Fernando $]$.

1996 Turismo actistico en Chichén Itzá, ponencia presentada en el 3er. Congreso Mexicano de Actistica. El documento está disponible en http:/www.gama. fime.uanl.mx/acustica/articulo/chichen.doc (consulta hecha el 7/12/2007).

Flores Dorantes, Felipe, y Lorenza Flores Garcia

1984 "Clasificación musical de silbatos prehispánicos mayas", Investigociones Rechentes en el Area Maya. XVI Mesa Redonda, tomo I: 439-443. Chiapas: Sociedad Mexicana de Antropologia.

Houston, Stephen, y Kart Taube

2000 "An Archaeology of the Senses: Perception and Cultural Expression in Ancient Mesoamerica", Cambridge Archaeological foumal, 10 (2) 261-294.

Kinsler, Lawrence E, y Austin R. Frey (eds.)

1950 Fundementals of Acoustics. Nueva York: John Wiley and Sons.

Lubman, David

1998 "Archaeological Acoustic Study of Chirped Echo from Mayan Pyramid at Chichen Itza", f. Acoust. Soc. Am., 104 [3].1763.

1999 "Singing stairs", Scl. News. 155: 44. Washington D.C.

1999b "Mayan Acoustics: Of Rainbows and Resplendent Quetzals", J. Acrust. Soc. Am. $106\langle 4\rangle, 2228$.

2002 "Rcoustical Features of Two Mayan Monuments at Chichen Itza: Accident of Design", J. Acoust. Soc. Amt, 112 (5), 2285.

Moore, 1: Richard

1990 Elements of Computer Music. Nueva Jersey, Estados Unidos: Prentice- Hall.

Muñoz Castillo, Fernando

2000 Teatro maya pentinsulo. Mérida: Ayuntamiento de Mérida iColección Capital Americana de la Cultura).

Ruz., Mario Humberto

1995 "Caracoles, dioses, santos y tambores. Expresiones musicales de los pueblos mayas", Dimensiein Antropológica, año 2, vol. 4: 37-86. México: INAH.

Stevenson. Robert

1968 Music in Aztec and Inca Terntory. Berkeley: University of Califomia Press. 
Van Kirk, Wayne

2002 "The Accidental (Acoustical) Tourist", J. Acoust. Soc. Amt., 112 (5), 2284.

Vela, David

1972 "Danzas y primeras manifestaciones del indigena maya-quiche", Anterica indigeno, vol. 32 (2): 515-521. México, Instituto Indigenista Interamericano.

Velázquez Cabrera, Roberto

2002 "Salbatos de Yaxchilán (Ranas de Barro)", ponencia en el First Pan-Americoni Iberion Meeting on Acoustics, Cancún, Quintana Roo.

Zalaquett Rock, Francisca A.

2006 "Estudio de las representaciones escénicas en los mayas del periodo Clasico: el Grupo Norte de Palenque y su significado social*. Tesis doctoral. México: UNAM, Facultad de Filosofia y Letras. 